Revue d'histoire de l'Amérique française

RBS REVUE D.HISTOIRE DE L'AMÉRIQUE FRANÇAISE

\title{
Normes de citations de données informatisées
}

\section{Jacques Rouillard}

Volume 47, numéro 2, automne 1993

URI : https://id.erudit.org/iderudit/305242ar

DOI : https://doi.org/10.7202/305242ar

Aller au sommaire du numéro

Éditeur(s)

Institut d'histoire de l'Amérique française

ISSN

0035-2357 (imprimé)

1492-1383 (numérique)

Découvrir la revue

Citer ce document

Rouillard, J. (1993). Normes de citations de données informatisées. Revue

d'histoire de l'Amérique française, 47(2), 306-308.

https://doi.org/10.7202/305242ar d'utilisation que vous pouvez consulter en ligne.

https://apropos.erudit.org/fr/usagers/politique-dutilisation/ 


\section{NORMES DE CITATIONS DE DONNÉES INFORMATISÉES}

Le Comité de rédaction soumet à l'attention de ses lecteurs les normes pour les citations de données informatisées proposées par le Comité canadien d'histoire et d'informatique. Nous invitons les lecteurs qui le souhaitent à nous soumettre leurs commentaires car nous pensons adopter ces normes pour la Revue d'histoire de l'Amérique française. Nous remercions José E. Igartua, membre de ce Comité, de nous avoir communiqué ces informations.

JACQUES ROUILLARD directeur

\section{I - OBJECTIFS}

Ces normes ont pour objet premier de définir pour les données informatisées des normes de citations équivalentes à celles qui régissent les sources traditionnelles, afin d'adapter l'appareil critique pratiqué par les historiens aux nouvelles modalités de la recherche historique.

Les normes ont également pour objectif de reconnaître le travail scientifique que constituent l'élaboration et la diffusion des bases de données à caractère historique, comme cela se pratique dans d'autres disciplines des sciences sociales.

Les normes ont enfin pour but d'aider les chercheurs à rencontrer les obligations faites par certains organismes subventionnaires (dont le Conseil de recherches en sciences humaines du Canada) de diffuser les données recueillies au moyen de leurs subventions.

\section{II - TROIS CAS D'ESPÈCE}

A - L'auteur utilise des bases de données constituées et diffusées par d'autres que lui (autres chercheurs, organismes de recherche, organismes à but lucratif).

Les citations de ces données doivent être rédigées selon le modèle fourni plus bas.

B - L'auteur utilise ses propres données, qu'il a rendu accessibles ou qu'il est prêt à rendre accessibles à d'autres chercheurs, aux conditions qu'il fixera, soit directement, soit par l'intermédiaire d'un organisme tiers 
(services d'archives traditionnels, services d'archives ordinolingues ou «data libraries», groupes de recherche ou autres organismes).

Le fait de rendre ses données accessibles leur donne le caractère d'une publication, et par conséquent les données ainsi «publiées» sont citées selon le modèle fourni plus bas.

Il est fortement conseillé aux chercheurs de confier à un organisme (département, centre de recherche, service d'archives ou autre) le rôle de producteur et de diffuseur des données, selon des modalités qui seront établies par les deux parties.

C - L'auteur utilise ses propres données informatisées d'une manière analogue à ses notes sur papier et n'a pas encouru d'obligation de diffusion.

L'auteur cite les sources originales utilisées pour constituer ses fichiers informatisés. Il explique, dans le texte de l'article ou en note, selon l'importance de la question dans sa démonstration, les transformations importantes qu'il a fait subir à ses données. Par exemple, on devrait expliquer comment sont définies les catégories professionnelles à l'intérieur desquelles on a regroupé des intitulés professionnels.

\section{III - NORMES DE CITATION ${ }^{1}$}

Dans la mesure du possible, les informations incluses dans la citation proviennent du document lui-même ou de la documentation qui l'accompagne.

1 - Auteur: citation selon la méthode usuelle

2 - Titre: titre du fichier de données ou de la base de données ${ }^{2}$

3 - Support informatique: indiquer entre crochets qu'il s'agit d'un support informatique. Par exemple,

[fichier d'ordinateur]

4 - Mention de responsabilité, le cas échéant

«La responsabilité dont il est question ici est celle des individus ou de la collectivité désigné(e) comme le chercheur principal, ou encore les autres parties ayant joué un rôle important tels que le ministère client, l'organisme de financement ou l'organisme de parrainage ${ }^{3}$ ».

1. Les normes de citations proposées ici reprennent les règles proposées par Terry Cook et al., Références aux documents d'archives: comment établir les références aux documents conservés aux Archives publiques du Canada (Archives publiques du Canada, 1983), 11-12. Voir aussi Danielle Thibault, Guide de rédaction bilbiographique (Ottawa, Bibliothèque nationale du Canada, 1989), 112-114. On peut aussi consulter les normes définies par l'American Sociological Review, ainsi que les pratiques de catalogage de la Canadian Union List of Machine Readable Data Files (CULDAT) constituée par Edward H. Hanis, telles que rapportées dans Edward H. Hanis, «Reference and User Guide for the CULDAT Information System», London (Ont.), 22 janvier 1990 (CULDAT est un projet commandité par la direction des archives gouvernementales des Archives nationales du Canada).

2. Une base de données est un ensemble de fichiers de données reliés entre eux par des liens logiques.

3. Terry Cook et al., Références aux documents d'archives, 11. 
5 - Édition, série ou version, si indiquée

6 - Lieu et date de production et nom du producteur

7 - Lieu et date de diffusion et nom du diffuseur, si différent de celui du producteur

8 - Collection, si indiquée

9 - Renseignements complémentaires optionnels:

a) Préciser le contenu entre crochets si ce n'est pas évident par le titre

b) Indiquer le type de support informatique entre crochets; par exemple, [base de données en direct]

[bande magnétique]

[disquette]

[CD-ROM]

c) Si la base de données est «vivante», sujette à modification, indiquer la date d'utilisation comme date de production

d) Mentionner les conditions d'accès lorsqu'il y a lieu

Quelques exemples:

Centre interuniversitaire de recherche sur les populations SOREP, Base de données SAGUENAY [fichier d'ordinateur], SOREP, Chicoutimi, Université du Québec à Chicoutimi, 1992, [base de données en direct] accessible pour fins de recherche, sujet à l'approbation du comité de déontologie de SOREP.

Base universitaire CANSIM [fichier d'ordinateur], Ottawa, Statistique Canada, 1992 [bande magnétique].

Science Citation Index [fichier d'ordinateur], Philadelphia, Institute for Scientific Information, 1989 [CD-ROM].

IGARTUA, José E., Base de données MEMBERS2 [liste de membres du Comité canadien d'histoire et d'informatique] [fichier d'ordinateur] Montréal, Département d'histoire, Université du Québec à Montréal, 1992, [base de données en direct] accessible auprès de l'auteur sur demande. 